\title{
Temperature Compensation for Transformer-type Transducer
}

\author{
Kanoknuch Songsuwankit, ${ }^{1}$ Vanchai Riewruja, ${ }^{1 *}$ Pakorn Watanachaturaporn, ${ }^{2}$ \\ Apinai Rerkratn, ${ }^{1}$ and Wandee Petchmaneelumka ${ }^{1}$ \\ ${ }^{1}$ Department of Instrumentation and Control Engineering, School of Engineering, \\ King Mongkut's Institute of Technology Ladkrabang, Bangkok 10520, Thailand \\ ${ }^{2}$ Department of Computer Engineering, School of Engineering, \\ King Mongkut's Institute of Technology Ladkrabang, Bangkok 10520, Thailand
}

(Received April 30, 2021; accepted July 9, 2021; online published November 16, 2021)

Keywords: transformer-type transducer, LVDT, resolver, temperature compensation technique, CCII, opamp

A novel technique to compensate the temperature effect of a transformer-type transducer is proposed in this paper. The effect of the ambient temperature on the transformer-type transducer is investigated from a primary-winding current. The advantage of the proposed technique is that the temperature effect is compensated without requiring a temperature sensor, making it suitable for applications in robotic and automation systems operated in harsh environments. The primary-winding current of the transducer is generated using a second-generation current conveyor (CCII). The excitation signal of the transformer-type transducer is driven by the CCII and the current flowing through the primary winding is transferred to an output signal of the CCII. The deviation of the primary-winding current due to the temperature effect is evaluated from the output signal of the CCII. The temperature effect on the transducer is manipulated by a closed-loop principle using a subtract-and-sum action instead of a traditional proportional-plusintegral action to eliminate the deviation of the primary-winding current. Therefore, the temperature effect on the transducer is compensated. A linear variable differential transformer (LVDT) is used to demonstrate the proposed technique, whose performance is discussed in detail and confirmed experimentally. All devices used in this experiment are commercially available. Experimental results show that the measured error of the output signal from the LVDT at $70{ }^{\circ} \mathrm{C}$ can be reduced from $6.2 \%$ without temperature compensation to $0.06 \%$ by using the proposed technique, which has the advantages of a low cost, simple configuration, and high performance.

\section{Introduction}

A transformer-type transducer is an inductive transducer that is widely used in the measurement of displacement, position, and speed. ${ }^{(1-3)}$ Two transducers are used in many industrial applications, linear variable differential transformers (LVDTs) and resolvers. The operation principle of transformer-type transducers is similar to that of electrical transformers.

*Corresponding author: e-mail: vanchai.ri@kmitl.ac.th

https://doi.org/10.18494/SAM.2021.3421 
Transformer-type transducers exhibit high reliability, high resolution, and robustness, making them suitable for application in measurement systems in harsh environments. Transformer-type transducers can be used in many applications such as the measurement of position and displacement in industry, as well as in robotics, aircraft, electric vehicles, and scientific equipment. ${ }^{(4-8)}$ Practically, transformer-type transducers require a sinusoidal signal as an excitation signal. The output signal of these transducers is in the form of amplitude modulation with suppressed carriers. ${ }^{(1,3)}$ Therefore, synchronous demodulation is required to obtain the position or displacement signal from the output signal of the transducer. ${ }^{(1,9-14)}$ Usually, traditional synchronous demodulators have a large response time and are inaccurate due to the large time constant of the low-pass filter used in them. The disadvantages mentioned above can be prevented using a peak-amplitude finder as proposed in the literature. ${ }^{(15-18)}$ The performance of the output signal from a transformer-type transducer is dependent on two factors, the structures of the transducer and signal conditioner. Many techniques to improve the structure of transducers have been proposed. ${ }^{(19-21)}$ However, these techniques require special customization of each transducer, making them expensive and not commercially available. Techniques for improving the performance of transducers by modifying the signal conditioner have been proposed. ${ }^{(22-26)}$ The variation of the ambient temperature is one of the major parameters reducing the accuracy of transducers through its effects on the mutual inductance and the resistance of the winding of the transducer structure. Increasing the ambient temperature will increase the resistance of the winding in the transducer. Also, the current flowing through the transducer is decreased, generating an error in the output signal of the transducer. Unfortunately, the above techniques of improving the accuracy of transducers do not compensate the temperature effect. The traditional approach to minimizing the temperature effect of transformer-type transducers is based on the inverse tangent of the ratio of two output signals. ${ }^{(1,3)}$ This approach provides a large error due to the non-ideal behaviors of the transducer and the divider used to calculate the ratio of the two output signals. Other techniques are based on the use of a temperature-sensitive device to compensate the temperature effect. ${ }^{(27-29)}$ However, the temperature-sensitive device requires similar behavior to the transducer, which is inconvenient and unsuitable for commercial transducers. Approaches using the output signal of a transformertype transducer to determine the temperature effect have recently been presented. ${ }^{(30,31)}$ However, these approaches are only suitable for transformer-type transducers used for linear measurement. On the basis of the transformer principle, the parameters of the secondary-winding side can be referred to the primary-winding side. ${ }^{(32)}$ Therefore, the error due to the temperature effect in the output signal of a transformer-type transducer can also be obtained from the primary-winding side. In this paper, the error extracted from the primary-winding side due to the temperature effect is obtained using a second-generation current conveyor (CCII). ${ }^{(33)}$ The compensation of the temperature effect exploits the subtract-and-sum topology in the closed-loop principle to minimize the size of the active device. The advantages of the proposed technique are its high performance and the reduced size of the device, making it suitable for robotic applications in harsh environments. 


\section{Principle}

The temperature effect on a transformer-type transducer can be obtained using the principle of the electrical transformer by referring to the parameters from the secondary-winding side to the primary-winding side. The simplified diagrams of transformer-type transducers are similar to that of an electrical transformer as shown in Fig. 1, where the secondary winding is terminated by the load resistance $R_{L}$. Usually, the primary winding of a transformer-type transducer requires the excitation signal $v_{e x}$ in the form of a sinusoidal signal such as $v_{e x}=V_{P} \sin \left(\omega_{e x} t\right)$, where $V_{P}$ is the peak amplitude and $\omega_{e x}$ is the excitation frequency. The variation of the ambient temperature affects the winding resistance of both the primary and secondary windings. An increase in the ambient temperature increases the resistance of both windings, which generates an error in the amplitude of the signal from the secondary winding. The deviation of the transducer parameters due to the temperature effect can be expressed in terms of the transformation ratio. From Fig. 1, the secondary-winding signal with a temperature effect can be expressed as

$$
v_{S}=(1-\alpha \Delta T) k_{T} V_{P} \sin \left(\omega_{e x} t\right)
$$

where $k_{T}$ and $\alpha$ are the transformation ratio and temperature coefficient of the transducer, respectively, and $\Delta T$ is the deviation of the ambient temperature from room temperature at 30 ${ }^{\circ} \mathrm{C}$. If an excitation voltage signal is applied to the primary winding, then the current flowing through the resistance $R_{L}$ of the secondary winding can be investigated from the primarywinding side. The peak-amplitude current of the secondary-winding side with reference to the primary-winding side can be obtained to achieve the temperature effect and can be expressed as

$$
i_{P}=\frac{(1-\alpha \Delta T) V_{P}}{\left|Z_{P S}\right|}=(1-\alpha \Delta T) k_{P S} V_{P}
$$

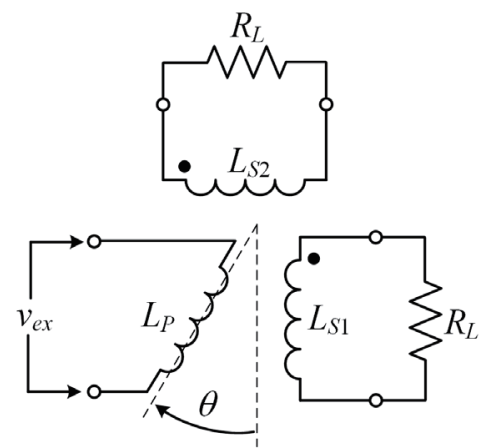

(a)

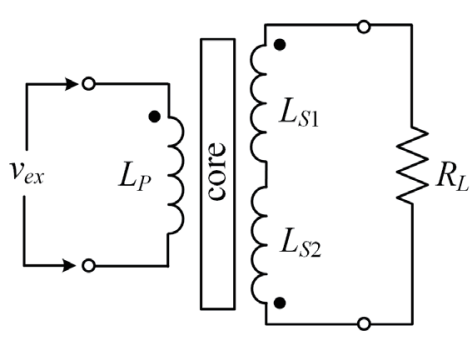

(b)

Fig. 1. Transformer-type transducers: (a) resolver and (b) LVDT. 
where $k_{P S}=1 / Z_{P S}$ and $Z_{P S}$ denotes the combination of the impedance from the primary side and the impedance referred from the secondary side to the primary side of the transformer-type transducer.

From Eq. (2), if the temperature effect of the primary-winding current $i_{P}$ is compensated, then the secondary-winding signal is simultaneously compensated. The temperature effect on the primary current $i_{P}$ can be achieved using the behavior of the CCII as shown in Fig. 2(a), where the primary-winding $L_{P}$ is driven by port $\mathrm{x}$ of the CCII. The behavior of the CCII is given by ${ }^{(33)}$

$$
\left[\begin{array}{l}
i_{y} \\
v_{x} \\
i_{z}
\end{array}\right]=\left[\begin{array}{lll}
0 & 0 & 0 \\
1 & 0 & 0 \\
0 & 1 & 0
\end{array}\right]\left[\begin{array}{l}
v_{y} \\
i_{x} \\
v_{z}
\end{array}\right] .
$$

From Eq. (3), the voltage signal $v_{x}$ and the current signal $i_{z}$ are set to equal the voltage signal $v_{y}$ and the current signal $i_{x}$, respectively. From Fig. 2(a), the primary current $i_{P}$ is achieved from port $\mathrm{x}$ and transferred to the current signal $i_{z}$ at port $\mathrm{z}, i_{z}=i_{P}$. The current signal $i_{z}$ is converted to the voltage signal $v_{z}$ using the resistance $R_{z}$ as follows:

$$
v_{z}=(1-\alpha \Delta T) k_{P S} R_{z} v_{e x}=(1-\alpha \Delta T) k_{R Z} v_{e x} .
$$

A block diagram representing the operation of the circuit in Fig. 2(a) with the temperature effect is shown in Fig. 2(b). The principle of the proposed technique of compensating the temperature effect is shown in Fig. 3(a), where $k_{P}$ and $G_{a}$ are the proportional gain and the phase-advance compensator, respectively.

The voltage signal $v_{z}$ containing the temperature effect is provided to determine the error signal $v_{e}$ by subtracting the input signal $v_{i n}$. The error signal $v_{e}$ is gained and summed with the input signal $v_{i n}$. The output signal $v_{S}$ of the sum-and-subtract topology in the dashed-line frame of Fig. 3(a) is used as the excitation signal $v_{e x}$. Usually, the error signal is reduced using an integral action in the feedback control system. Unfortunately, the integral action in the feedback

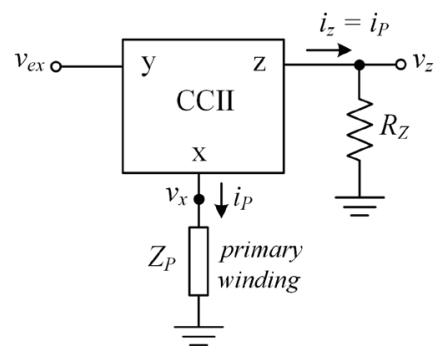

(a)

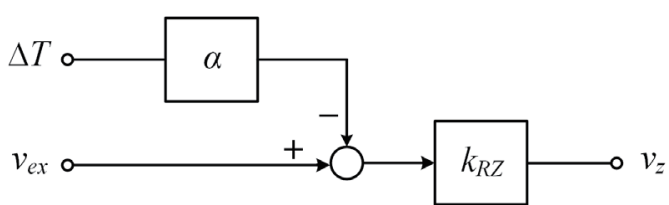

(b)

Fig. 2. Primary-winding current extraction using CCII: (a) current diagram and (b) block diagram. 


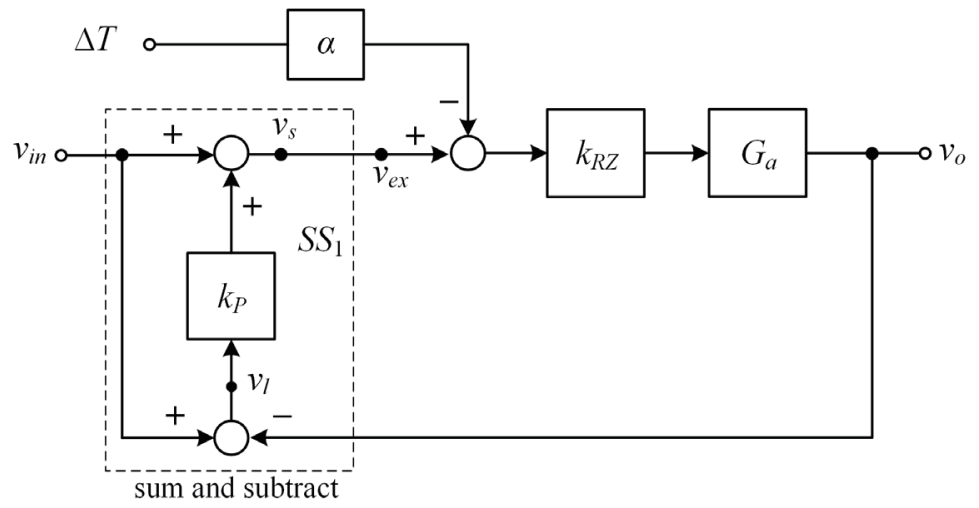

(a)

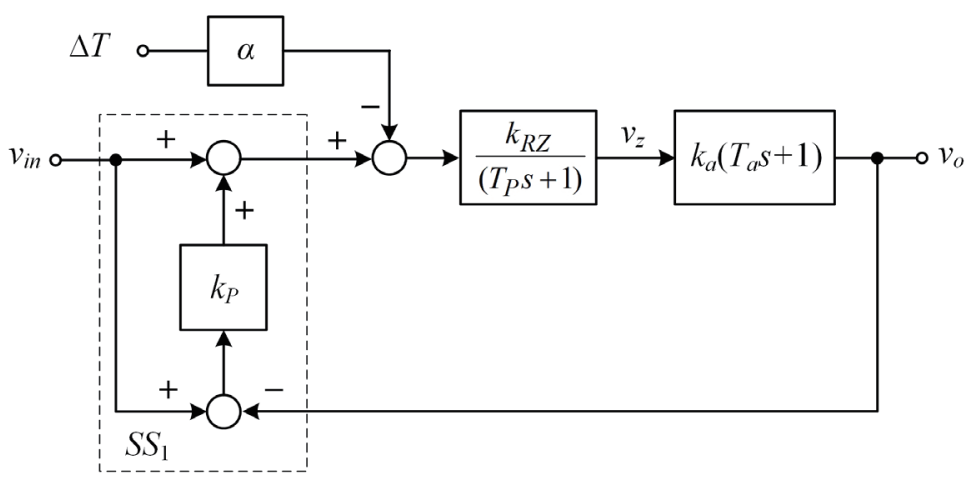

(b)

Fig. 3. Proposed technique: (a) principle and (b) block diagram with dynamic behavior.

control system is only suitable for an input signal in the form of the Heaviside function. If the input signal is sinusoidal, then a phase shift of the signal occurs in the feedback loop. Therefore, the use of the integral action in the feedback control system will cause the dominant pole of the system to be close to the instability condition. The sum-and-subtract topology does not require a time-dependent device. Therefore, the instability problem can be avoided. From Fig. 3(a), the phase-advance compensator $G_{a}$ is provided to compensate the phase lag and scaling factor for the signal from the transfer characteristic of the transducer in Eq. (4). The closed-loop transfer function of Fig. 3(a) is given by

$$
v_{o}=\frac{\left(1+k_{P}\right) k_{R Z} G_{a}}{1+k_{P} k_{R Z} G_{a}} v_{i n}-\frac{\alpha k_{R Z} G_{a}}{1+k_{P} k_{R Z} G_{a}} \Delta T .
$$

The primary impedance $Z_{P S}$ in Eq. (2) is composed of the winding resistances and the winding inductances of both the primary and secondary sides. Thus, a phase lag $\theta_{P}$ occurred for the voltage signal $v_{z}$ relative to the excitation signal $v_{e x}$. The transfer characteristic of the transformer-type transducer together with the CCII in Fig. 2(a) can be approximated by the firstorder expression 


$$
\frac{v_{z}(s)}{v_{y}(s)}=\frac{(1-\alpha \Delta T) k_{R Z}}{\left(T_{P} s+1\right)}
$$

where $T_{P}$ is the time constant of the transducer. $T_{P}$ can be determined from the phase lag $\theta_{P}$ as

$$
T_{P}=\frac{\tan \left|\theta_{P}\right|}{\omega_{e x}} .
$$

The phase-advance compensator $G_{a}$ is provided to compensate the phase lag of the transducer. In practice, the phase shift $\theta_{a}$ of the phase-advance compensator is set to equal $\left|\theta_{P}\right|$ and the time constant $T_{a}$ is set to equal the time constant $T_{P}$. Then, the transfer characteristic of the phaseadvance compensator $G_{a}(\mathrm{~s})$ can be expressed as

$$
G_{a}(s)=k_{a}\left(T_{a} s+1\right)
$$

where $k_{a}$ and $T_{a}$ are the compensation gain and time constant of the compensator, respectively. $T_{a}$ is determined from the phase shift $\theta_{a}=\left|\theta_{P}\right|$. Therefore, the time constants $T_{a}$ and $T_{P}$ are set to be equal. The dynamic behavior of Fig. 3(a) is shown in Fig. 3(b), and the closed-loop transfer function can be expressed as

$$
v_{o}(s)=\frac{\frac{\left(1+k_{p}\right)\left(T_{a} s+1\right) k_{R Z} k_{a}}{\left(T_{P} s+1\right)}}{1+\left[\frac{k_{p} k_{R Z} k_{a}\left(T_{a} s+1\right)}{\left(T_{P} s+1\right)}\right]} v_{i n}(s)-\frac{\frac{\alpha\left(T_{a} s+1\right) k_{P} k_{R Z} k_{a}}{\left(T_{P} s+1\right)}}{1+\left[\frac{k_{p} k_{R Z} k_{a}\left(T_{a} s+1\right)}{\left(T_{P} s+1\right)}\right]} \Delta T .
$$

If $T_{P}$ and $T_{a}$ are set to be equal, then Eq. (9) can be rewritten as

$$
v_{o}(s)=\frac{1}{\left(1+k_{P} k_{R Z} k_{a}\right)}\left[\left(1+k_{P}\right) k_{R Z} k_{a} v_{i n}(s)-\alpha k_{R Z} k_{a} \Delta T\right] .
$$

Practically, the parameters $k_{R Z} k_{a}=1$ and $k_{P} \gg 1$ are set to minimize the error due to the temperature effect. The magnitude of Eq. (10) for $v_{i n}=V_{P} \sin \left(\omega_{e x} t\right)$ can be approximated as

$$
\left|v_{o}(j \omega)\right|=\left[v_{i n}(j \omega)+\frac{\alpha}{k_{P}} \Delta T\right]
$$

It can be seen that the temperature effect of the transformer-type transducer in the square brackets of Eq. (11) can be minimized with a large proportional gain $k_{p}$. 


\section{Circuit Description}

The circuit diagram of the proposed principle in Fig. 3(b) is shown in Fig. 4. The operation of the circuit in Fig. 4 is explained as follows. The opamp $A_{1}$ and resistors $R_{f 1}$ and $R_{f 2}$ function as the sum and subtract operations. The voltage signal $v_{e x}$ can be expressed as

$$
v_{e x}=\frac{R_{f 2}}{R_{f 1}}\left(v_{i n}-v_{o}\right)+v_{o}=k_{P}\left(v_{i n}-v_{o}\right)+v_{o}
$$

The voltage signal $v_{e x}$ in Eq. (12) is consistent with the sum and subtract in the dashed-line frame of Fig. 3(a). The voltage signal $v_{e x}$ is applied to port y of the CCII as the excitation signal of the transformer-type transducer.

From Eq. (3), the voltage signal $v_{x}$ at port $\mathrm{x}$ of the CCII is forced to equal the voltage signal $v_{e x}$ at port $\mathrm{y}$, then the current signal $i_{P}$ or the magnitude of the primary-winding current of the transformer-type transducer is determined as $i_{P}=v_{e x}|| Z_{P S} \mid=k_{P S} v_{e x}$. The current signal $i_{P}$ of the transducer is transferred to port $\mathrm{z}$ of the CCII and converted to the voltage signal $v_{z}$ by the resistor $R_{Z}$. The phase of the voltage signal $v_{z}$ with respect to the voltage signal $v_{\text {in }}$ is led by the phase-advance compensator formed by the opamp $A_{2}$, the resistors $R_{C 1}$ and $R_{C 2}$, and the capacitor $C_{C}$. The transfer characteristic of the phase-advance compensator can be expressed as

$$
\frac{v_{o}(s)}{v_{z}(s)}=\left(1+\frac{R_{C 2}}{R_{C 1}}\right)\left[\frac{R_{C 1} R_{C 2} C_{C}}{\left(R_{C 1}+R_{C 2}\right)} s+1\right]=k_{a}\left(T_{a} s+1\right) .
$$

From Eq. (13), the phase lead $\theta_{a}$ is given by

$$
\theta_{a}=\tanh ^{-1}\left(T_{a} \omega_{e x}\right)
$$

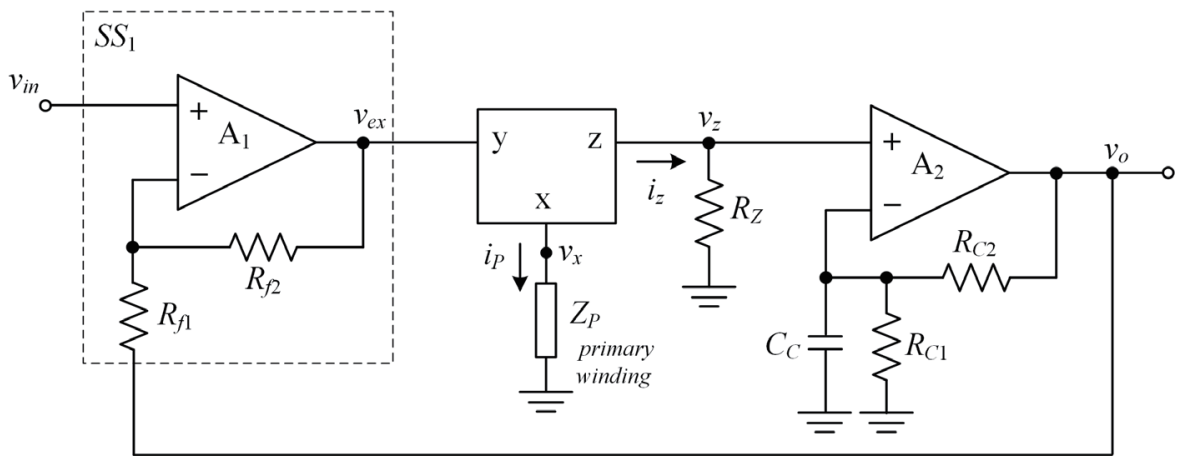

Fig. 4. Proposed circuit. 
From the circuit in Fig. 4 , if $R_{Z}\left(R_{C 1}+R_{C 2}\right)=R_{C 1}\left|Z_{P S}\right|$, then the transfer characteristic $v_{o}(s) / v_{i n}(s)$ can be written as

$$
v_{o}(s)=\left(1+\frac{R_{f 2}}{R_{f 1}}\right) v_{i n}(s)-\frac{R_{f 1}}{R_{f 2}} \alpha \Delta T .
$$

For $k_{P}=R_{f 2} / R_{f 1}$, Eq. (15) can be rewritten as

$$
v_{o}(s)=\left(1+k_{P}\right) v_{i n}(s)-\frac{\alpha \Delta T}{k_{P}}
$$

If the proportional gain $k_{p} \gg 1$ or $R_{f 2} \gg R_{f 1}$ and $s=j \omega$ are assigned, then the magnitude of Eq. (16) is given by

$$
\left|v_{o}(j \omega)\right|=\left|v_{i n}(j \omega)\right|-\frac{R_{f 1}}{R_{f 2}} \alpha \Delta T=\left|v_{i n}(j \omega)\right|-\frac{\alpha \Delta T}{k_{P}}
$$

It can be seen that the variation of the ambient temperature $\Delta T$ is minimized with a large proportional gain or $R_{f 1} \ll<R_{f 2}$ without a stability problem.

\section{Circuit Performance}

The performance of the proposed circuit in Fig. 4 deviates from the expected value owing to the non-ideal characteristic of the devices used in the circuit. The tolerance of the resistors and the dynamic behavior of the active devices used in the proposed circuit affect the stability and the circuit operation. Three factors contribute to the error in the voltage signal $v_{o}$. Firstly, the tolerance of the resistors $R_{f 1}$ and $R_{f 2}$ generates an error in the proportional gain $k_{P}$. The voltage signal $v_{o}$ with the error $\varepsilon_{1}$ can be approximated as

$$
v_{o}(s)=\left[1+k_{P}\left(1+2 \delta_{1}\right)\right] v_{i n}(s)-\frac{\left(1+2 \delta_{1}\right) \alpha \Delta T}{k_{P}},
$$

where $\delta_{1}$ denotes the tolerance of the resistors $R_{f 1}$ and $R_{f 2}$. The error of the proportional gain $k_{P}$ can be avoided by changing the resistor $R_{f 2}$ to a variable resistor and fine-tuning the variable resistor to an appropriate value. Secondly, the tolerance $\delta_{2}$ of the resistors $R_{C 1}$ and $R_{C 2}$ generates errors in the phase lead $\theta_{a}$ and gain $k_{a}$ in Eqs. (13) and (14). The transfer characteristic of the phase-advance compensator in Eq. (13) can be rewritten as

$$
\frac{v_{o}(s)}{v_{z}(s)}=k_{a}\left(1+\varepsilon_{1}\right)\left[T_{a}\left(1+\varepsilon_{2}\right) s+1\right] \text { for } \varepsilon_{1}=\frac{2 \delta_{2} R_{C 2}}{\left(R_{C 1}+R_{C 2}\right)} \text { and } \varepsilon_{2}=\frac{\delta_{2}\left(R_{C 2}-R_{C 1}\right)}{\left(R_{C 1}+R_{C 2}\right)}
$$


where $\delta_{2}$ denotes the tolerance of the resistors $R_{C 1}$ and $R_{C 2}$. If the resistances of $R_{C 1}$ and $R_{C 2}$ are set to be equal, than the error $\varepsilon_{2}$ can be eliminated. Also, the error $\varepsilon_{1}$ is equal to $\delta_{2}$ for the resistances $R_{C 1}=R_{C 2}$. Practically, the resistors used in the proposed circuit are selected for their tolerance of less than $0.1 \%$, i.e., $\delta_{1}$ and $\delta_{2}$ are less than $1 \times 10^{-3}$. Therefore, the error $\varepsilon_{1}$ is very small and can be neglected. Thirdly, the dynamic behaviors of the opamps and the CCII, exhibited in the term of the dominant pole, provide the phase delay for the signal in the circuit. Therefore, the stability of the circuit operation is deteriorated. The single-pole expression of the opamps $A_{1}$ and $A_{2}$ and the CCII are included in the block diagram in Fig. 3(b) as shown in Fig. 5. The phase lag $\theta_{P}$ due to the structure of the transformer-type transducer is compensated by the phase-advance compensator. Thus, the transfer characteristic of Fig. 5 including the dynamic behaviors of the opamps and the CCII can be expressed as

$$
v_{o}(s)=\frac{\frac{\left(1+k_{P}\right) k_{P S} k_{z} k_{a}}{\left(T_{1} s+1\right)\left(T_{2} s+1\right)\left(T_{3} s+1\right)}}{1+\frac{k_{P} k_{P S} k_{z} k_{a}}{\left(T_{1} s+1\right)\left(T_{2} s+1\right)\left(T_{3} s+1\right)}} v_{i n}(s)-\frac{\frac{\alpha k_{z} k_{a}}{\left(T_{1} s+1\right)\left(T_{2} s+1\right)\left(T_{3} s+1\right)}}{1+\frac{k_{P} k_{P S} k_{z} k_{a}}{\left(T_{1} s+1\right)\left(T_{2} s+1\right)\left(T_{3} s+1\right)}} \Delta T,
$$

where $T_{2}, T_{1}$, and $T_{3}$ denote the time constant of the CCII and the time constants of the opamps $A_{1}$ and $A_{2}$, respectively. The circuit operation of the CCII is in the current mode, which provides a fast response. Therefore, the time constant $T_{2}$ of the CCII in Eq. (20) is very small compared with the time constants $T_{1}$ and $T_{3}$ and can be neglected. The corner frequencies $\omega_{1}$ and $\omega_{3}$ from the time constants $T_{1}$ and $T_{3}$ can be approximated as

$$
\omega_{1}=\frac{G B P_{1}}{\left(1+k_{P}\right)}
$$

and

$$
\omega_{3}=\sqrt{\frac{G B P_{2}}{k_{a} T_{a}}},
$$

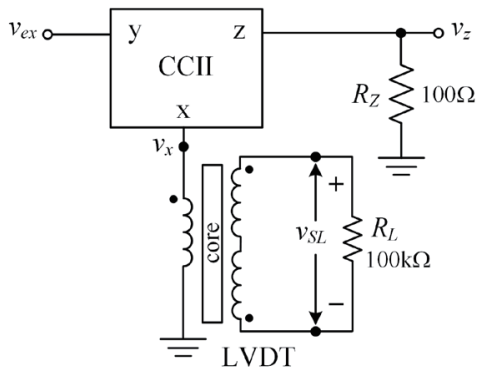

(a)

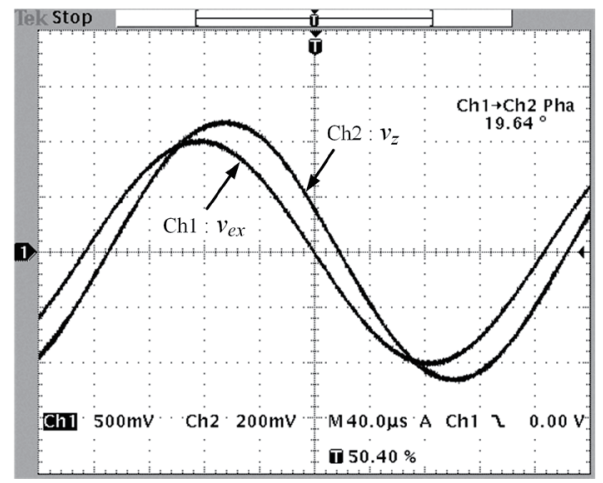

(b)

Fig. 5. Tested circuit for LVDT: (a) circuit diagram and (b) measured result. 
where $G B P_{1}$ and $G B P_{2}$ are the gain bandwidth products of the opamps $A_{1}$ and $A_{2}$, respectively. From Eq. (21), the corner frequency $\omega_{1}$ is inversely proportional to the gain $k_{P}$. The phase shift $\theta_{1}$ due to the corner frequency $\omega_{1}$ can be expressed as

$$
\theta_{1}=-\tan ^{-1}\left(\frac{\omega_{e x}\left(1+k_{P}\right)}{G B P_{1}}\right)
$$

From Eqs. (21) and (23), the high value of the gain $k_{P}$ causes the corner frequency $\omega_{1}$ to be close to the excitation frequency $\omega_{e x}$, which increases the phase shift $\theta_{1}$. Also, the compensation gain $k_{a}$ reduces the corner frequency $\omega_{3}$ to approach the excitation frequency $\omega_{e x}$. The phase shift $\theta_{3}$ of the corner frequency $\omega_{3}$ can be expressed as

$$
\theta_{3}=-\tan ^{-1}\left(\sqrt{\frac{\omega_{e x}^{2} k_{a} T_{a}}{G B P_{2}}}\right) .
$$

From Eq. (24), the high value of the gain $k_{a}$ increases the phase shift $\theta_{3}$. The increase in the phase shifts $\theta_{1}$ and $\theta_{3}$ causes the unstable operation of the proposed circuit. These phase shifts can be canceled by increasing the phase lead of the phase-advance compensator. The phase lead $\theta_{a}$ generated by the phase-advance compensator to minimize the effect of the phase shifts $\theta_{P}, \theta_{1}$, and $\theta_{3}$ can be rewritten as

$$
\theta_{a}=\theta_{P}+\theta_{1}+\theta_{3}
$$

Practically, the corner frequencies $\omega_{1}$ and $\omega_{3}$ are set to be much greater than the excitation frequency $\omega_{e x}, \omega_{1} \gg 10 \omega_{e x}$ and $\omega_{3} \gg 10 \omega_{e x}$, to reduce the phase shift due to these corner frequencies. The maximum values of the proportional gain $k_{P}$ and the compensation gain $k_{a}$ can be expressed as

$$
\begin{aligned}
& k_{P}=\frac{G B P_{1}}{10 \omega_{e x}}-1, \\
& k_{a}=\frac{G B P_{2}}{100 \omega_{e x}^{2} T_{a}} .
\end{aligned}
$$

From Eq. (27), the compensation gain $k_{a}$ is usually set to a small value of about $k_{a}<5$. Then the time constant $T_{3}$ can also be disregarded. From Eqs. (17) and (26), a high gain $k_{P}$ is provided to minimize the temperature effect. In contrast, the proportional gain $k_{P}$ is a major factor contributing to the unstable operation of the proposed circuit. If $G B P_{1}=25.13 \mathrm{Mrad} / \mathrm{s}$ and $\omega_{e x}=21.99 \mathrm{krad} / \mathrm{s}$, then the proportional gain is $k_{P}=113.27$. It can be seen that the temperature effect in Eq. (17) can be reduced more than 100-fold. 


\section{Experimental Results}

To demonstrate the performance of the proposed technique, the circuit in Fig. 4 is breadboarded. All passive and active devices used in the circuit are commercially available. The active devices used in this paper are AD844 for the CCII and LF353, consisting of two opamps in the same package, for the opamps $A_{1}$ and $A_{2}$, where the GBP of LF353 is $25.13 \mathrm{Mrad} / \mathrm{s}$. All resistors have a tolerance of $0.1 \%$. A power supply of $\pm 8 \mathrm{~V}$ is set. The input signal $v_{\text {in }}$ is a $3 \mathrm{kHz}$ sinusoidal wave with $2 \mathrm{~V}$ peak-to-peak amplitude. The proportional gain $k_{P}$ is 100 . Therefore, the resistors $R_{f 1}$ and $R_{f 2}$ are set to 2 and $200 \mathrm{k} \Omega$, respectively. The transformer-type transducer selected for this experiment is a commercial LVDT with $\pm 12.5 \mathrm{~mm}$ stroke range and sensitivity of $34.5 \mathrm{mV} / \mathrm{mm} / \mathrm{V}^{(15)}$ The tested circuit shown in Fig. 5(a) is used to determine the parameters of the LVDT.

The load resistor for the secondary winding of the LVDT is set as $100 \mathrm{k} \Omega$ and the converted resistance $R_{Z}$ of $100 \Omega$ is connected to port z. The measured result in Fig. 5(b) shows the primary-winding current transferred to port z of the CCII and converted to the voltage signal $v_{z}$, where the excitation signal $v_{e x}$ is set as a $3 \mathrm{kHz}$ sinusoidal wave with $2 \mathrm{~V}$ peak-to-peak amplitude. The phase shift $\theta_{P}$ of the current flowing through the primary winding is measured as $-19.64^{\circ}$. From Fig. 5(b), the magnitude of the impedance $\left|Z_{P S}\right|$ is determined as $212.76 \Omega$, which is the impedance of the primary-winding side included with the parameters referred from the secondary-winding side to the primary-winding side. From Eq. (7), the phase shift $\theta_{P}$ corresponds to the time constant $T_{P}$ of $18.93 \mu \mathrm{s}$. The resistors $R_{C 1}$ and $R_{C 2}$ are set to be equal and a capacitor $C_{C}$ of $0.01 \mu \mathrm{F}$ is chosen. The time constant $T_{a}$ is set to equal the time constant $T_{P}$. Thus, the resistors $R_{C 1}$ and $R_{C 2}$ are determined from Eq. (13) as $3.786 \mathrm{k} \Omega$. Practically, the resistances $R_{C 1}$ and $R_{C 2}$ are chosen as $3.8 \mathrm{k} \Omega$. To satisfy the condition $R_{Z}\left(R_{C 1}+R_{C 2}\right)=R_{C 1}\left|Z_{P S}\right|$, the resistance $R_{z}$ is set to equal $\left|Z_{P S}\right| / 2(106.38 \Omega)$. A variable resistor that can be adjusted to satisfy this condition is chosen for the resistor $R_{z}$. The ambient temperature in this experiment is varied from room temperature of $25^{\circ} \mathrm{C}$ to $70{ }^{\circ} \mathrm{C}$. Also, the core position of the LVDT is set to $+10 \mathrm{~mm}$ to obtain a voltage signal $v_{S L}$ of $345 \mathrm{mV}$. Figure 6(a) shows the measured primarywinding current represented by the voltage signal $v_{z}$ and the output signal $v_{S L}$ of the secondary winding of the LVDT without temperature compensation. From Fig. 6(a), the temperature coefficient $\alpha$ of the LVDT is measured and determined from the voltage signal $v_{Z}$ as -0.65 $\mathrm{mV} /{ }^{\circ} \mathrm{C}$ for the $2 \mathrm{~V}$ peak-to-peak amplitude of the voltage signal $v_{e x}$. The percentage error of the measured results can be expressed as

$$
\text { percentage error }(\%)=\frac{\mid \text { actual value }- \text { measured value } \mid}{\text { actual value }} \times 100 \% \text {. }
$$

The percentage errors of the measured results in Fig. 6(a) are shown in Fig. 6(b). It can be seen that the maximum percentage errors of the primary current and the output signal $v_{S L}$ of the LVDT at $70{ }^{\circ} \mathrm{C}$ are about 6.2 and $6.76 \%$, respectively. The measured results and the percentage errors for the voltage signal $v_{z}$ and the output signal $v_{S L}$ of the LVDT with the compensation technique using the proposed scheme are shown in Fig. 6(b). From Fig. 6(b), the primary- 


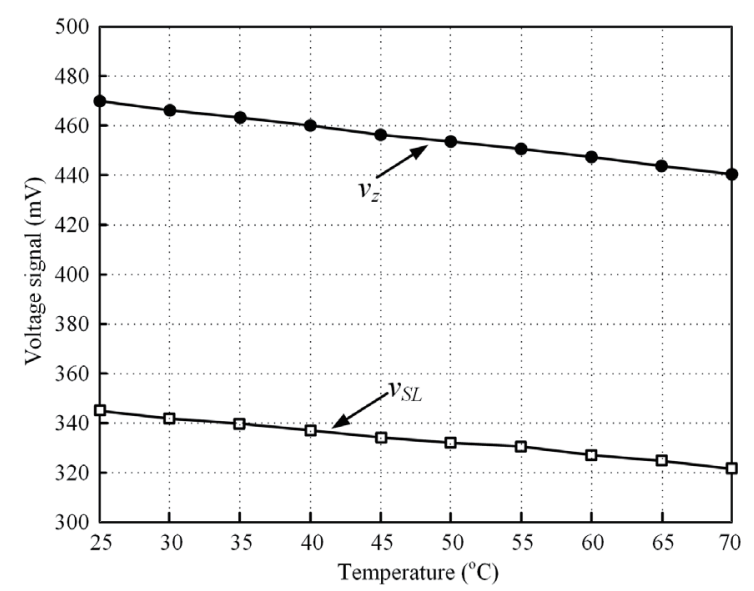

(a)

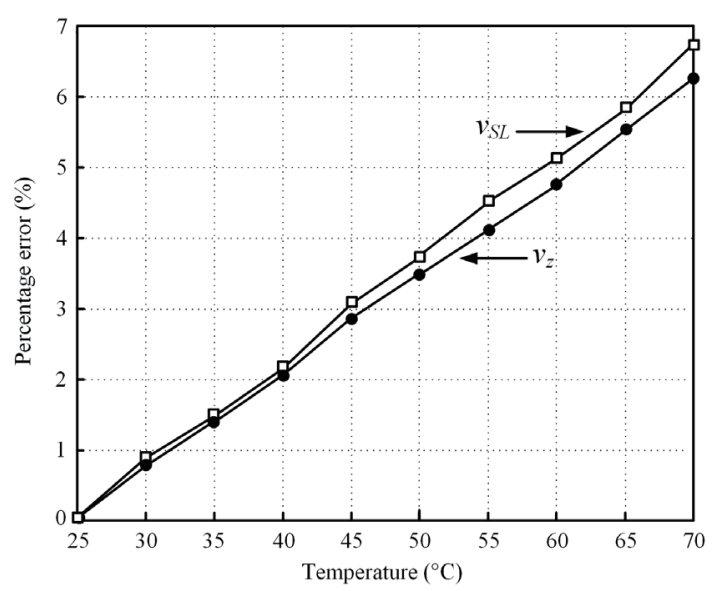

(b)

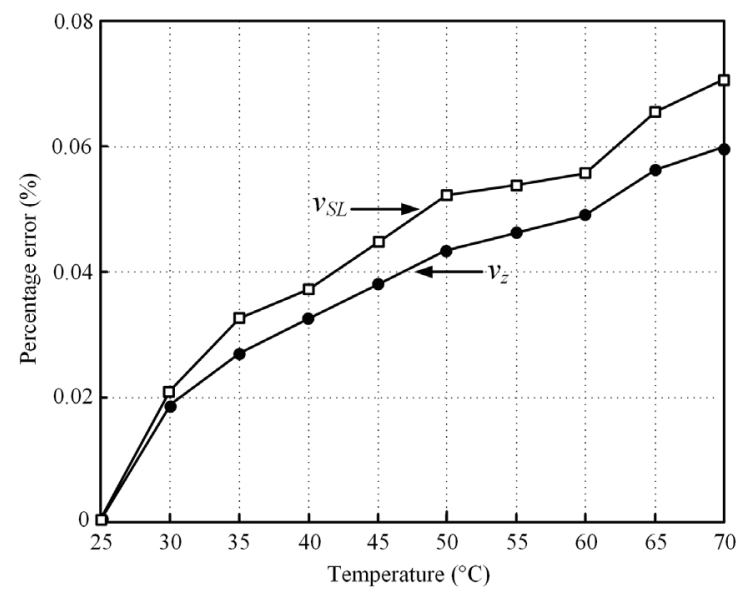

(c)

Fig. 6. Experimental results: (a) results without compensation of signal $v_{z}$, (b) results without compensation, and (c) results with compensation.

winding current represented by the voltage signal $v_{z}$ and the output signal $v_{S L}$ of the LVDT exhibit errors of about 0.06 and $0.07 \%$, respectively, at $70{ }^{\circ} \mathrm{C}$. It can be clearly seen that the proposed technique can reduce the temperature effect more than 100 -fold.

\section{Conclusions}

A simple technique to compensate the temperature effect for a transformer-type transducer has been introduced. The proposed technique does not require a temperature-sensitive device to compensate the temperature effect for the transducer. The primary-winding current containing the parameters referred from the secondary-winding side is used to achieve the temperature effect. The technique is based on the use of the CCII behavior to extract the primary-winding current. This paper considers an LVDT as an example of a transformer-type transducer. The 
experimental results confirm that the temperature effect on the output signal of the LVDT is reduced more than 100-fold. The merits of the proposed circuit are its simple configuration, small size, low cost, and ability to be directly embedded in a transducer without disturbing the configuration of the signal conditioner system.

\section{References}

1 R. Pallas-Areny and J. G. Webster: Sensor and Signal Condition (Wiley, New York, 2001) p. 229.

2 S. Cetinkunt: Mechatronics (Wiley, New York, 2006) p. 227.

3 H. K. P. Neubert: Instrument Transducers - An Introduction to Their Performance and Design (Oxford, New York, 1975) p. 204.

4 S. Lee and W. Kim: IEEE Trans. Control Syst. Tech. 18 (2010) 859. https://doi.org/10.1109/TCST.2009.2030413

5 M. Felix, A. Lizarraga, A. Islas, and A. Gonzales: 36th Conf. IEEE Industrial Electronics Society (IECON, 2010) 1769. https://doi.org/10.1109/IECON.2010.5675411.

6 G. Chen, B. Zhang, P. Liu, and H. Ding: IEEE Sens. J. 15 (2015) 2248. https://doi.org/10.1109/ JSEN.2014.2364610

7 J. Lee, K. C. Lee, S. Cho, and S. H. Sim: Sensors 17 (2017) 2317. https://doi.org/10.3390/s17102317

8 Z. Zhang, S. H. Rau, W. J. Lee, T. Gammon, and B. C. Johnson: IEEE Trans. Ind. Appl. 52 (2016) 5241. https:// doi.org/10.1109/ESW.2016.7499707

9 A. Ota, W. Petchmaneelumka, A. Rerkratn, and V. Riewruja: 32nd JSST Int. Conf. Simulation Technology (2013).

10 J. Tongcharoen, W. Petchmaneelumka, and V. Riewruja: 15th Int. Conf. Control, Automation and Systems (ICCAS, 2015) 1699. https://doi.org/10.1109/ICCAS.2015.7364630

11 A. Drumea, A. Vasile, M. Comes, and M. Blejan: 1st Electronic System-Integration Technology Conf. (ESTC, 2006) 629. https://doi.org/10.1109/ESTC.2006.280070

12 R. M. Ford, R. S. Weissbach, and D. R. Loker: IEEE Trans. Instrum. Meas 3 (2000) 1448. https://doi. org/10.1109/IMTC.2000.848714

13 R. Casanella, O. Casas, and R. Pallas-Areny: Meas. Sci. Technol. 16 (2005) 1637. https://doi.org/10.1088/09570233/16/8/014

14 C. S. Koukourlis, V. K. Trigonidis, and J. N. Sahalos: IEEE Trans. Instrum. Meas 42 (1993) 926. https://doi. org/10.1109/19.252529

15 W. Petchmaneelumka, K. Songsuwankit, and V. Riewruja: I.R.E.E. 11 (2016) 340. https://doi.org/10.15866/iree. v11i3.8906

16 W. Petchmaneelumka, K. Songsuwankit, A. Rerkratn, and V. Riewruja: 3rd Int. Conf. Control, Automation and Robotics (ICCAR, 2017) 758. https://doi.org/10.1109/ICCAR.2017.7942799

17 W. Petchmaneelumka, K. Songsuwankit, and V. Riewruja: 9th Int. Conf. Computer and Automation Engineering (ICCAE, 2017) 193. https://doi.org/10.1145/3057039.3057103

18 W. Petchmaneelumka, P. Mano, K. Songsuwankit, and V. Riewruja: Int. J. Electron. 105 (2018) 1520. https:// doi.org/10.1080/00207217.2018.1461250

19 K. Ara: IEEE Trans. Instrum. Meas. 21 (1972) 249. https://doi.org/10.1109/TIM.1972.4314011

20 S. C. Saxena and S. B. L. Seksena: IEEE Trans. Inst. Meas. 38 (1989) 748. https://doi.org/10.1109/19.32186

21 R. Alipour-Sarabi, Z. Nasiri-Gheidari, F. Tootoonchian, and H. Oraee: IEEE Sens. J. 17 (2017) 7877. https://doi. org/10.1109/JSEN.2017.2761796

22 W. Petchmaneelumka, W. Koodtalang, and V. Riewruja: IEEE Sens. J. 19 (2019) 5045. https://doi.org/10.1109/ JSEN.2019.2902879

23 W. Petchmaneelumka, K. Songsuwankit, J. Tongcharoen, and V. Riewruja; Sens. Mater. 32 (2020) 475. https:// doi.org/10.18494/SAM.2020.2407

24 R. Liu and H. Bu: Sens. Transducers 160 (2013) 68.

25 K. Wang and Z. Wu: IEEE Trans. Ind. Elec. 67 (2020) 4867. https://doi.org/10.1109/TIE.2019.2931250

26 L. Pecly, R. Schindeler, D. Cleveland, and K. Hashtrudi-Zaad: IEEE Trans. Instrum. Meas. 66 (2017) 2917. https://doi.org/10.1109/TIM.2017.2714378

27 T. S. Smith: U.S. Patent US 5087866 A (1991).

28 A. Masi, S. Danzeca, R. Losito, P. Peronnard, R. Secondo, and G. Spiezia: Nucl. Instrum. Method Phys. Res. 745 (2014) 73. https://doi.org/10.1016/j.nima.2014.01.054

29 Q. Li and F. Ding: Sens. Actuators 122 (2005) 83. https://doi.org/10.1016/j.sna.2005.04.008 
30 W. Petchmaneelumka, A. Rerkratn, A. Luangpol, and V. Riewruja: J. Circ. Syst. Comp. 27 (2018) 1850182. https://doi.org/10.1142/S0218126618501827

31 W. Petchmaneelumka, P. Mano, and V. Riewruja: Sens. Mater. 30 (2018) 2171. https://doi.org/10.18494/ SAM.2018.1816

32 J. A. Melkebeek: Electrical Machines and Drives - Fundamentals and Advanced Modelling (Springer, Switzerland, 2018) p. 10.

33 V. Riewruja and W. Petchmaneelumka: AEU Int. J. Electron. Commun. 62 (2008) 725. https://doi. org/10.1016/j.aeue.2007.09.007 